\title{
BMJ Open Association between socioeconomic status and dental caries among Chinese preschool children: a cross-sectional national study
}

\author{
Tingting Zhang (D , ${ }^{1}$ Jialan Hong, ${ }^{2}$ Xueting Yu, ${ }^{1}$ Qiulin Liu, ${ }^{1}$ Andi Li, ${ }^{1}$ Zhijing Wu, ${ }^{1}$ \\ Xiaojuan Zeng
}

To cite: Zhang T, Hong J, Yu X, et al. Association between socioeconomic status and dental caries among Chinese preschool children: a cross-sectional national study. BMJ Open 2021;11:e042908. doi:10.1136/ bmjopen-2020-042908

- Prepublication history for this paper is available online. To view these files, please visit the journal online (http://dx.doi. org/10.1136/bmjopen-2020042908).

Received 18 July 2020 Revised 27 April 2021 Accepted 04 May 2021

Check for updates

(C) Author(s) (or their employer(s)) 2021. Re-use permitted under CC BY-NC. No commercial re-use. See rights and permissions. Published by BMJ.

${ }^{1}$ Department of Dental Public Health, College \& Hospital of Stomatology of Guangxi Medical University, Nanning, China

${ }^{2}$ Department of Epidemiology \& Public Health, University College London, London, UK

Correspondence to Professor Xiaojuan Zeng; xiaojuan.zeng@qq.com

\section{ABSTRACT}

Objectives Socioeconomic inequalities in oral health are often neglected in oral health promotion. This crosssectional study assessed the association between dental caries and socioeconomic status (SES) among preschool children in China.

Design Cross-sectional study.

Setting Data from the Fourth National Oral Health Survey of China (2015), comprising of 40360 children aged 3-5 years was used.

Methods Dental caries indicators including prevalence of dental caries, dental pain experience and number of decayed, missing and filling teeth (dmft). SES indicators included parental education and household income. The associations between SES and dental caries were analysed by using negative binomial regression or Poisson regression models according to data distribution. Relative and absolute inequalities in dental caries were quantified by using the Relative Index of Inequality (RII) and Slope Index of Inequality (SII), respectively.

Results There were significant associations between SES and prevalence of dental caries and dmft $(p<0.001)$. Children from lower educated (RII 1.36, 95\% Cl 1.3 to 1.43; SII $0.97,95 \% \mathrm{Cl} 0.81$ to 1.13 ) and lower household income (RII 1.17, $95 \% \mathrm{Cl} 1.11$ to 1.24 ; SII $0.55,95 \% \mathrm{Cl}$ 0.35 to 0.75 ) families had higher dmft than those from well-educated and most affluent families. Relative and absolute inequalities in dental caries were larger in urban areas by household income, and in rural areas by parental education.

Conclusions Association between dental caries and SES was demonstrated and socioeconomic inequalities in dental caries existed among Chinese preschool children.

\section{INTRODUCTION}

Currently, dental caries is still the greatest global oral health burden with 532 million children affected worldwide. ${ }^{1}$ Dental caries not only post a threat on health and quality of life but also impose a substantial economic burden on the society. ${ }^{2}$ Although WHO found that the prevalence of dental caries has been declined over the past decade, the declining trend in dental caries was evident
Strengths and limitations of this study

- The first study to quantify socioeconomic inequalities in dental caries among Chinese preschool children using relative and absolute inequality regression.

- The data were from a relatively large cross-sectional national study.

- Cross-sectional nature of the study design precluding inference about causality.

in high-income countries but was nonsignificant in low-income and middle-income countries, ${ }^{34}$ even the prevalence of dental caries has increased in some low-income and middle-income countries, suggesting that oral health inequalities remain across countries.

An individual's socioeconomic status (SES) is one of the most important determinants in children's oral health, ${ }^{5}$ and evidence has been found that children with low SES, including low household income, low mother's education and living in socially disadvantaged families, were more likely to have higher prevalence of dental caries and greater dental pain experience. ${ }^{6-8}$ In India, a lagged analysis of a structural equation modelling showed that SES contribute to oral health status indirectly. ${ }^{9}$ Poor SES can have a deleterious impact on child oral health as a result. Socioeconomic inequality in child dental caries is a great concern in many countries. ${ }^{7810}$ Considering children's critical role in ensuring the well-being of oral health inequality, it is important to explore the oral health in children.

China is the world's most populous country, having 1.4 billion people. ${ }^{11}$ China has been undergoing rapid economic developments while also experiencing a processing of increasing inequalities in health. ${ }^{12}$ For example, Chinese children from rural areas 
or poorer families are more likely to be stunted than those from urban areas or wealthier families. ${ }^{13} 14$ The inequalities in children's oral health were also observed in China ${ }^{15} 16$, and childhood oral health inequalities can persist into adulthood, irrespective of later changes in social position. ${ }^{17}$ However, few studies have explored the association between SES and oral health in Chinese preschool children. ${ }^{18}$ Hence, additional research to improve current understanding of socioeconomic inequalities in oral health in preschool children of China is needed.

This study was to explore the association between SES and dental caries, and evaluated the socioeconomic inequalities in dental caries among children aged 3-5 years around the mainland of China.

\section{METHODS}

\section{Data source}

We used data from the Fourth National Oral Health Survey of China conducted in 2015, which was based on a nationally representative sample of 40360 children aged 3-5 years old, providing information on individual oral health status, sociodemographic data and general health status. As previously described,$^{20}$ a multistage cluster sampling method was used. Written consent was obtained by parents of each child to participate in the study.

Dental examination was completed by trained and calibrated dentists during the national survey. Those with kappa values higher than 0.8 for the decayed, missing and filling teeth $(\mathrm{dmft})$ index were qualified. Dental caries diagnostic criteria were adopted according to the WHO recommendation. ${ }^{21}$ Socioeconomic information from the children's families was obtained by structured questionnaire finished by their parents.

\section{Dependent variables}

The three main dependent variables of dental caries status were (1) prevalence of dental caries, (2) dental pain experience ('yes' or 'no'), defined as having toothache in the last 12 months, reported by the parents and (3) dmft (count variable), the number of dmft.

\section{Independent variables}

Parental education and household income were considered as SES indicators. Parental education was grouped into three categories: low level (secondary school degree or below), middle level (high school degree) and high level (college degree or above) according to the Chinese education system. Household income in the study year (2015) was categorised into five groups according to National Income Quintiles of China: lowest ( $\leq$ US\$4000/ year), low (US\$4000-US\$9000/year), middle (US\$9000US\$15 000/year), high(US\$15000-US\$20 000/year), highest(>US\$20000/year).

\section{Covariates}

Age, gender, ethnic (Han/other ethnics), place of residence (urban/rural) and region (east/central/west) as well as parent-reported child general health (good or better, fair or less) were considered as covariates.

\section{Statistical analysis}

Data were analysed using STATA MP V.16.0 (StataCorp). Descriptive results were conducted in order to identify the main patterns of data. Proportional differences between different groups were compared by using $\chi^{2}$ tests. Continuous data lack of normal distribution was analysed using Mann-Whitney test (two groups) or Kruskal-Wallis test (more than two groups).

Multiple imputation was carried out for incomplete data in parental education and household income, which were 10 and 15010 , respectively. Overall distribution of available values was used to determine the values to be imputed, ${ }^{22}$ and 40 imputed datasets were generated according to the proportion of missing data, which was at least equal to the percentage of incomplete data. ${ }^{23}$ The collinearity between income and education was assessed. Their variance inflation factors were both less than 10 , indicating these two SES indicators cannot be considered as a linear combination of other independent variables.

Poisson regression was used to assess the associations between SES indicators and prevalence of dental caries or dental pain. ${ }^{24}$ Since the proportion of 'zero' caries counts was only $37.5 \%,{ }^{25}$ a negative binomial regression model was used to assess the association between SES indicators and the $\log \mathrm{dmft}$. ORs for Poisson regression and incidence rate ratio (IRR) for negative binomial regression with $95 \%$ CIs were reported. Estimates were significantly different from the reference if its $95 \%$ CIs do not include 1. Crude model and adjusted model were built. Adjusted model further take consideration of the covariates.

Considering the social structure of the population, the Relative Index of Inequality (RII) and Slope Index of Inequality (SII) were used to assess relative and absolute inequalities, respectively. ${ }^{26}$ By disposing the SES indicators as a continuous variable, RII and SII use all available data and are not limited to comparisons of extreme groups, and finally result into two different types of measures of socioeconomic inequalities in health, which are relative and absolute. The SII estimated the absolute predicted difference in caries experience between the highest and lowest SES, interpreted as the difference in predicted health rates at the two extremes of the socioeconomic spectrum, and RII is their ratio. Values of RII $>1$ or SII $>0$ signify existence of an SES gradient in oral health, and higher the score the greater the magnitude of the inequity. Considering the survey weights and missing data, all the above covariates were included in the models. The ridit score for estimating SII and RII was calculated. ${ }^{27}$ Using the ridit score and continuous caries experience measurements, the ratio of the mean by Poisson regression was considered as RII and the beta coefficient by linear regression was considered as SII. The ridit score, 


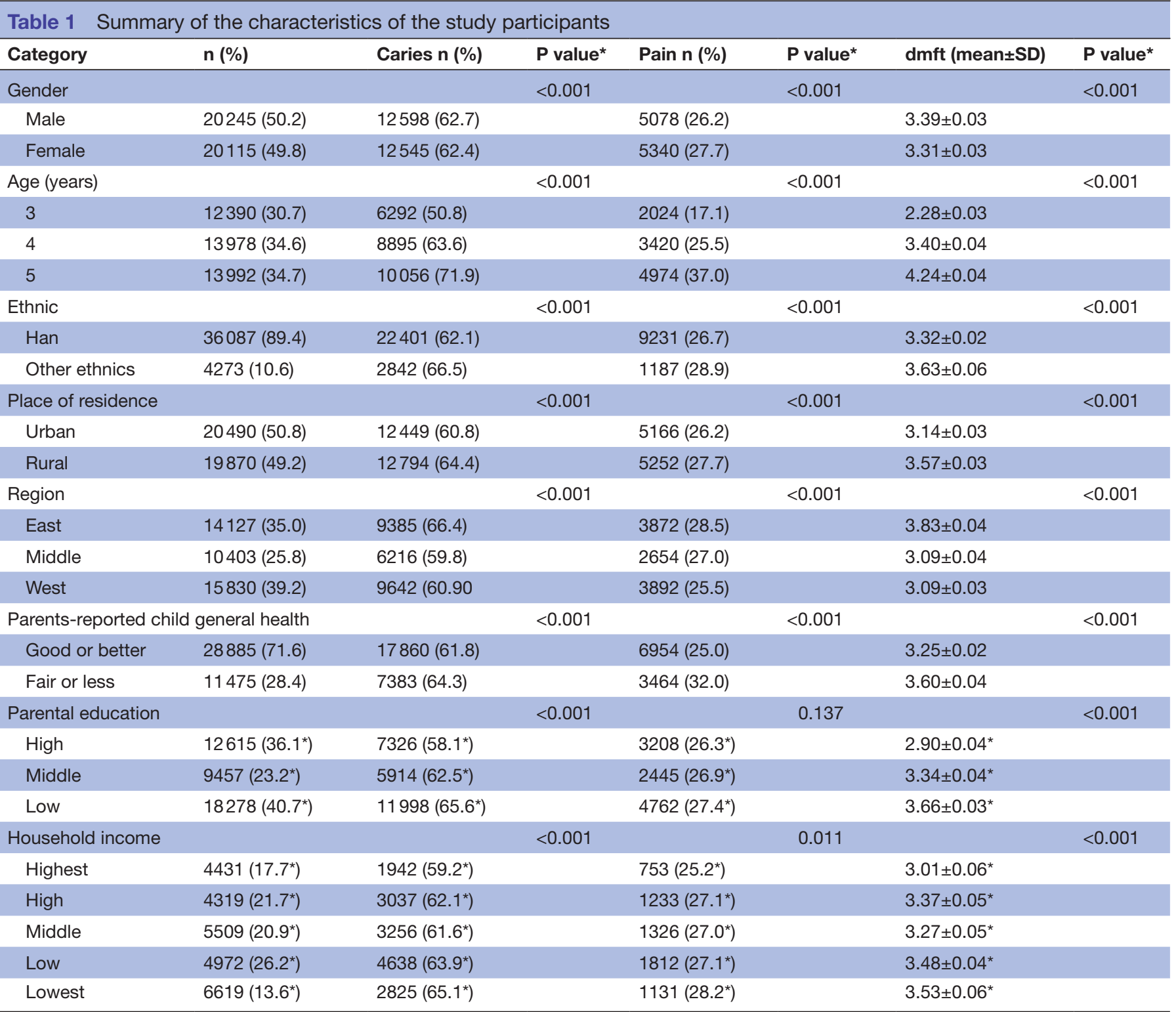

*Proportions and $p$ values are presented after multiple imputation for parental education and household income. $\mathrm{dmft}$, decayed, missing and filling teeth.

RII and SII were calculated for each of the 40 datasets and RII and SII were integrated.

Taking into account sampling method and the post stratification, all models were survey-weighted. Analyses were also conducted stratifying by urban areas and rural areas.

\section{Patient and public involvement}

Patients and the public were not involved in developing the research question, study design or outcome measures. While direct dissemination of study results has not been planned, they will be communicated through our institutional media services.

\section{RESULTS}

Of the 40360 children in the study, $50.2 \%$ were boys and $49.8 \%$ were girls. Sociodemographic information was summarised in table 1 . In addition, the prevalence of dental caries and dental pain, and mean dmft in rural areas were higher than those in urban areas, and there was an increasing trend with age, parental education and household income (table 1).

The prevalence of dental caries and dental pain was $62.5 \%$ and $26.9 \%$, respectively, and the mean $\mathrm{dmft}$ was $3.35 \pm 0.02$ (table 2 ).

There were significant associations between oral health and both SES indicators $(\mathrm{p}<0.001)$. After adjusting for gender, age, ethnic, region, place of residence and parent-reported child general health, the existence of social gradients in dental caries indicators was confirmed, with the exceptions of dental pain. Figure 1 shows that children from middle and low parental education group had higher dmft (IRR 1.13, 95\% CI 1.09 to 1.17; and IRR 
Table 2 Basic characteristics of dental caries indicators in the study participants

\begin{tabular}{lll}
\hline & \multicolumn{2}{l}{ Overall } \\
\cline { 2 - 3 } Category & $\mathbf{n}$ & $\%$ \\
\hline Caries & 25243 & 62.5 \\
Dental pain & 10418 & 26.9 \\
dmft (mean \pm SD) & $3.35 \pm 0.02$ & \\
\hline
\end{tabular}

dmft, decayed, missing and filling teeth.

$1.20,95 \%$ CI 1.17 to 1.24 , respectively). This pattern was also observed for prevalence of dental caries by parental education. Additionally, there was a gradient in the association between household income and prevalence of dental caries and dmft (figure 1).

RII and SII estimates showed significant relative and absolute inequalities for oral health and SES indicators except for dental pain experience. We observed higher dmft among children in the lowest household income families (IRR 1.16, 95\% CI1.10 to 1.23) than those from the highest household income families (figure 1), with this being reflected significantly in the relative and absolute index of inequality (RII 1.17, 95\% CI 1.11 to 1.24 and SII $0.55,95 \%$ CI 0.35 to 0.75 ) (figure 2), representing an excess of $1.17 \mathrm{dmft}$ and 55 more children with $\mathrm{dmft}$ per 100 children in the lowest household income group compared with the highest one, respectively. Similarly, relative inequalities were as well as larger in prevalence of dental caries and dmft by parental education (RII 1.17, $95 \%$ CI 1.13 to 1.21 and RII $1.36,95 \%$ CI 1.30 to 1.43 , respectively). Significant absolute and relative inequalities in dental pain were also observed when stratified by place of residence. In rural areas, inequalities in dental caries in favour of those with lower household income and lower parental education. However, parental education was only significantly associated with prevalence of dental pain in rural areas (RII $0.87,95 \%$ CI 0.79 to 0.95 and SII $-0.05,95 \%$ CI -0.08 to -0.03 ). Meanwhile, our findings revealed that inequalities were larger in rural areas by parental education, while inequalities related to household income were larger in urban areas (figure 2).

\section{DISCUSSION}

In general, we identified a social gradient in oral health of children, with lower SES being associated with a higher risk of dental caries and dental pain experience. In urban areas, a positive gradient was observed with higher parental education being associated with higher dental pain experience. Different from children in urban areas whose inequalities in dental caries were larger by

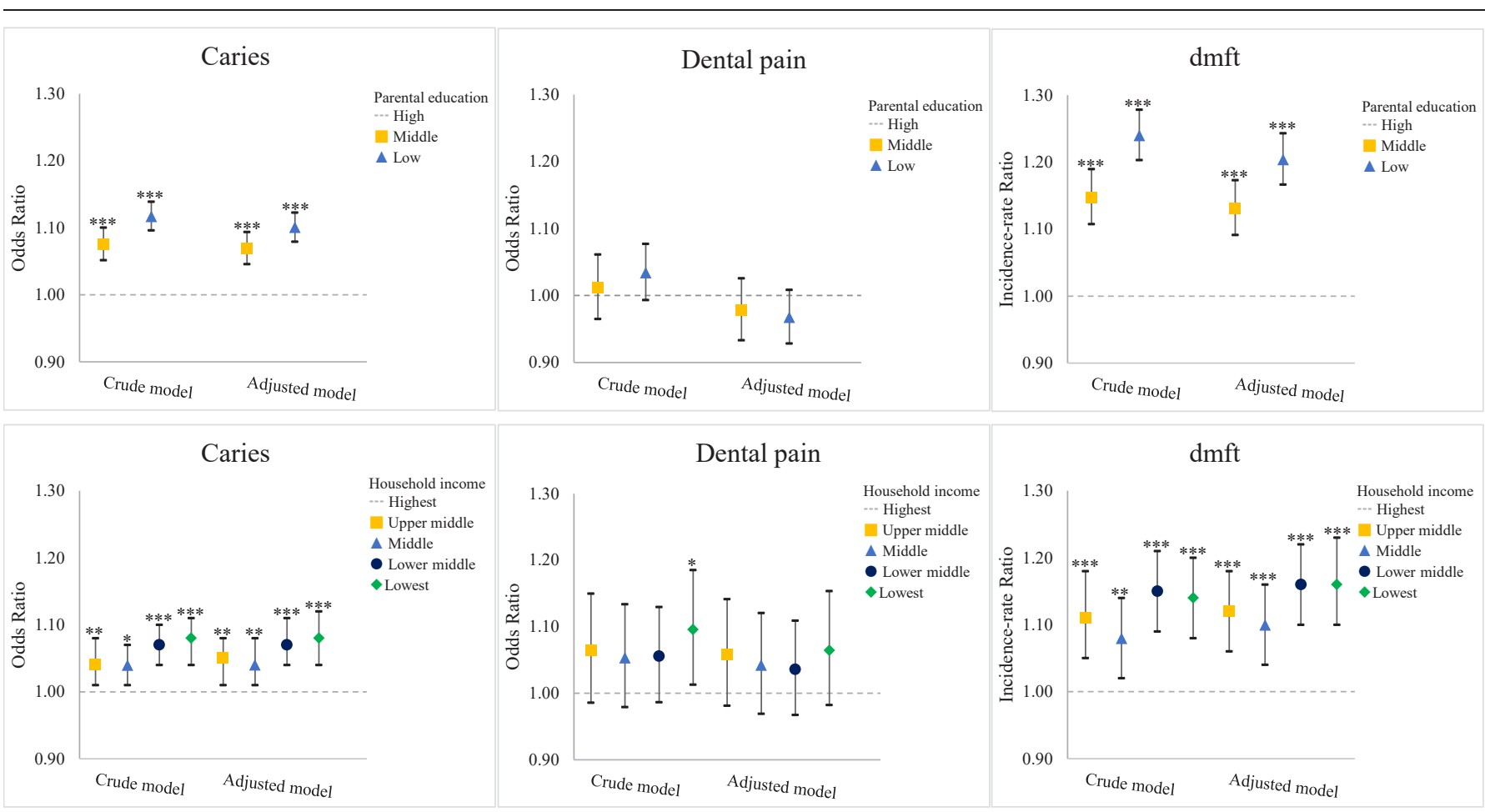

Figure 1 OR, incidence rate ratio (IRR) stratified by parental education and household income. OR, IRR and $95 \% \mathrm{Cls}$ by parental education and household income are presented as well as the level of significance. Crude model: each SES measure (parental education and household income) and outcomes (dental caries, dental pain and dmft). Adjusted model: adjusted for age, gender, ethnic, place of residence, region and parent-reported child general health. All models are weighted. ${ }^{*} \mathrm{P}<0.05$, ${ }^{* *} \mathrm{p}<0.01,{ }^{* * *} \mathrm{p}<0.001$. dmft, decayed, missing and filling teeth; SES, socioeconomic status. 

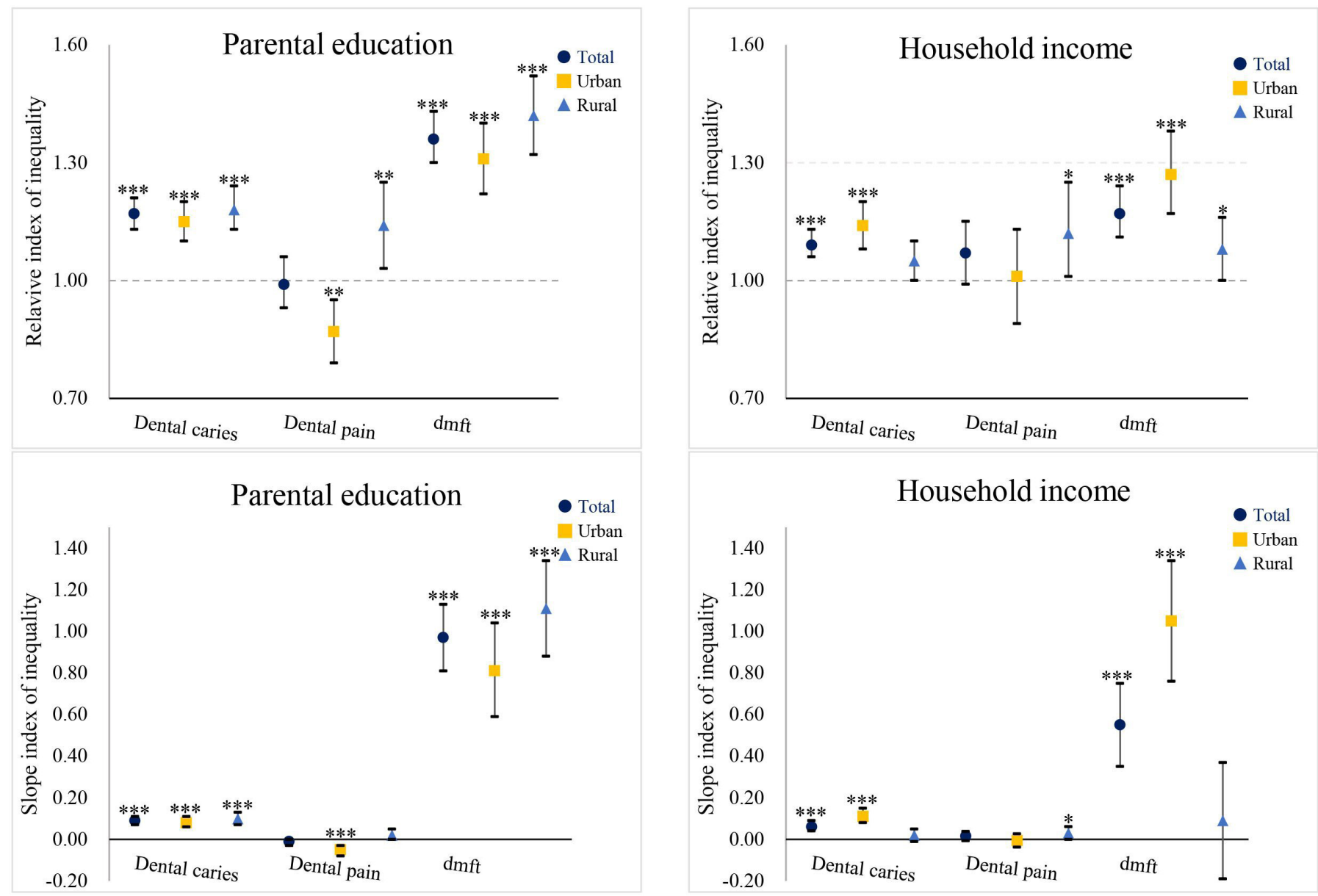

Figure 2 Relative Index of Inequality and Slope Index of Inequality for urban and rural area by parental education and household income. Estimates and $95 \% \mathrm{Cls}$ are presented as well as the level of significance, adjusted by age, gender, ethnic, region and parent-reported child general health. All models are weighted. ${ }^{\star} \mathrm{P}<0.05,{ }^{\star *} \mathrm{p}<0.01,{ }^{\star \star *} \mathrm{p}<0.001$. dmft, decayed, missing and filling teeth.

household income, inequalities in dental caries of children in rural areas were more affected by parental education. This characteristic should be considered in future oral health promotion programmes.

Parental education and household income were obvious markers relating to oral health in children, with lower parental education and household income being significantly associated with higher prevalence of dental caries and higher dmft in this study. This finding was in agreement with an earlier dental health inequality studies. ${ }^{628}$ There was some evidence showed that children from lower SES families suffer from more severe dental pain, higher prevalence of dental caries and dental flourosis. ${ }^{29-31}$ Among 3-year-old Japanese children, higher prevalence of dental caries was associated with lower level of parental education. ${ }^{28} \mathrm{~A}$ cross-sectional study in Australia showed that parental education with higher level were significantly inversely associated with $\mathrm{dmft}$ of children aged 4-13 years old. ${ }^{32}$ On the other hand, no association was observed between parental education and caries experience in Chinese ${ }^{33}$ and Mongolian children, ${ }^{8}$ which may be due to small sample size and the time of data collection.
This study also revealed household income as a traditional SES indicator of children, affected the distribution of caries experience. Evidence from a recent study confirmed that household income was one of the strongest factors related to oral health. ${ }^{34}$ A cohort study on trends in oral health from a life course data in Hong Kong suggested that household income had an effect on children's oral health status. ${ }^{35}$ Significant inverse associations between household income and dental caries were also observed in Chinese ${ }^{16}$, American, ${ }^{36}$ Japanese, ${ }^{28}$ Australian $^{632}$ and Mongolian ${ }^{8}$ children.

Our findings also revealed that inequality by parental education was existed in lower parental education in rural areas. And children in rural areas also had higher $\mathrm{dmft}$ and prevalence of dental caries than those in urban areas, which keeping with the trend of a former study in China. ${ }^{33}$ Our finding is also consistent with a Thailand study which examined the time trends in dental caries among children and indicated the prevalence of dental caries was higher for the children who lived in rural areas. ${ }^{37}$ However, from the perspective of household income, relative and absolute inequalities were larger in urban areas in the results. We found that parental education was positively associated 
with dental pain experience in urban areas. This might be explained by the fact that the neglect of discomfort and pain in children from low parent educated groups, with proportion of high educated parents being larger in urban areas, and larger inequalities by household income in urban areas. Health services utilisation is as well as a potential factor accounting for the large inequalities in health between urban and rural residents in China. ${ }^{38} 39$ Utilisation of dental services had a positive impact on the caries experience in children and adolescents. ${ }^{35}$

Parental SES might influence child oral health through oral health practice, knowledge and attitude. ${ }^{40}$ Parents of higher education visited a dentist more frequently not only when their children had dental pain, but also to bring their children in for preventive checkups and learn oral health knowledge. ${ }^{41}{ }^{42}$ Meanwhile, education is a primary determinant of a person's labour market position on the other hand, which in turn influences income, housing and other material resources. And higher income promotes improved living conditions, such as safe housing, ability to preferentially attend public dental services and receive oral health advice compared with those from lower income. ${ }^{5}$

However, parental education and household income are difficult to modify in the short term. Therefore, strategies must be developed to improve oral health of children, facilitate parental knowledge and promote preventive tools. Our findings would advance the argument for oral health promotion initiatives that engage parents of children very early. For example, the positive effect of increased household income and high parental education on child health implies that government provide health service targeting the poor and the illiteracy may be an effective way to improve the oral health of children from low SES families, and public welfare programmes should focus on rural areas, or considering the importance of child oral health in future life quality, which implies a potential increasing oral health education in such an inequality in oral health of children. Oral health inequalities are not unconquerable but need government support. For example, socioeconomic inequalities in oral health of children were less conspicuous in areas with water fluoridation compared with non-fluoridated places in Australia. ${ }^{43}$ Policies targeting poverty to reduce socioeconomic inequalities may be successful as well as the interventions in health utilisation and oral health services. $^{44}{ }^{45}$ Interprofessional collaboration between professional dentists, non-dentistry professionals and fellow-health professionals should be established to jointly provide services aiming at low SES groups at the same time. ${ }^{46}$

\section{Strength and limitation}

A major strength of our study was the Fourth National Oral Health Survey of China use of a relatively large and representative sample of children, which ensured study results are likely to be generalisable across the mainland of China children. And it was the first study to measure inequalities in child oral health by using SII and RII in China. The study findings should be considered with number of limitations. The study design was the crosssectional nature precluding inference about causality. We were not able to examine how socioeconomic inequalities in oral health changed as children grew into adolescents. Longitudinal studies of the oral health of representative samples of Chinese children are rare, and that will provide stronger evidence of the potential causal pathways underlying oral health inequalities as further longitudinal data become available.

\section{CONCLUSIONS}

Children from the lower SES families were more likely to have dental caries. Furthermore, significant inequalities can be found at a very early age.

Acknowledgements The authors are grateful to the study participants whose data has made this study possible. This study is conducted in partnership between 35 colleges and universities, Center of Disease Control (CDC) of Chinese Health Commission of the People's Republic of China, Chinese Stomatological Association.

Contributors All authors meet the ICMJE authorship criteria. TZ, XZ, JH and XY conceived the study and developed the analysis strategy. TZ, JH and XY carried out the analysis. TZ drafted the manuscript. QL, AL and ZW critically reviewed the drafts, gave text suggestions and approved the final manuscript.

Funding This study was supported by 'Scientific Research Fund of National Health Commission of the People's Republic of China (201502002)'.

Competing interests None declared.

Patient and public involvement Patients and/or the public were not involved in the design, or conduct, or reporting, or dissemination plans of this research.

Patient consent for publication Not required.

Ethics approval Ethical approval was obtained from the Ethics Committee of Chinese Stomatological Association (approval no. 2014-003).

Provenance and peer review Not commissioned; externally peer reviewed.

Data availability statement Data are available on reasonable request. Consent has not been obtained to share the data publicly.However, data may be accessed on contacting the corresponding author. The same principle applies for statistical analysis scripts.

Open access This is an open access article distributed in accordance with the Creative Commons Attribution Non Commercial (CC BY-NC 4.0) license, which permits others to distribute, remix, adapt, build upon this work non-commercially, and license their derivative works on different terms, provided the original work is properly cited, appropriate credit is given, any changes made indicated, and the use is non-commercial. See: http://creativecommons.org/licenses/by-nc/4.0/.

ORCID iD

Tingting Zhang http://orcid.org/0000-0002-3669-3174

\section{REFERENCES}

1 GBD 2017 Disease and Injury Incidence and Prevalence Collaborators. Global, regional, and national incidence, prevalence, and years lived with disability for 354 diseases and injuries for 195 countries and territories, 1990-2017: a systematic analysis for the global burden of disease study 2017. Lancet 2018;392:1789-858.

2 Righolt AJ, Jevdjevic M, Marcenes W, et al. Global-, regional-, and country-level economic impacts of dental diseases in 2015. J Dent Res 2018;97:501-7.

3 Lagerweij MD, van Loveren C. Declining caries trends: are we satisfied? Curr Oral Health Rep 2015;2:212-7.

4 Frencken JE, Sharma P, Stenhouse L, et al. Global epidemiology of dental caries and severe periodontitis - a comprehensive review. J Clin Periodontol 2017;44:S94-105. 
5 Fisher-Owens SA, Gansky SA, Platt LJ, et al. Influences on children's oral health: a conceptual model. Pediatrics 2007;120:e510-20.

6 Kilpatrick NM, Neumann A, Lucas N, et al. Oral health inequalities in a national sample of Australian children aged 2-3 and 6-7 years. Aust Dent J 2012;57:38-44.

7 Rouxel P, Chandola T. Socioeconomic and ethnic inequalities in oral health among children and adolescents living in England, Wales and Northern Ireland. Community Dent Oral Epidemiol 2018;46:426-34.

8 Chinzorig T, Aida J, Cooray $\mathrm{U}$, et al. Inequalities in caries experience among mongolian children. Int J Environ Res Public Health 2019;16 doi:10.3390/ijerph16203892

9 Gupta E, Robinson PG, Marya CM, et al. Oral health inequalities: relationships between environmental and individual factors. J Dent Res 2015;94:1362-8. doi:10.1177/0022034515592880

$10 \mathrm{Ha} \mathrm{DH}$, Do LG, Roberts-Thomson K, et al. Risk indicators for untreated dental decay among Indigenous Australian children. Community Dent Oral Epidemiol 2019;47:316-23.

11 Statistics NBo. Statistical bulletin of the People's Republic of China on national economic and social development in 2019, 2020.

12 Fang P, Dong S, Xiao J, et al. Regional inequality in health and its determinants: evidence from China. Health Policy 2010;94:14-25.

13 Liu H, Fang H, Zhao Z. Urban-rural disparities of child health and nutritional status in China from 1989 to 2006. Econ Hum Biol 2013;11:294-309.

14 Chen Y, Lei X, Zhou L-A. Does raising family income cause better child health? Evidence from China. Econ Dev Cult Change 2017;65:495-520.

15 Quan JK, Wang XZ, Sun XY, et al. Permanent teeth caries status of 12- to 15-year-olds in China: findings from the 4th national oral health survey. Chin J Dent Res 2018;21:181-93.

16 Chen X, Ye W, Zhan JY, et al. Periodontal status of Chinese adolescents: findings from the 4th national oral health survey. Chin $J$ Dent Res 2018;21:195-203.

17 Thomson WM, Poulton R, Milne BJ, et al. Socioeconomic inequalities in oral health in childhood and adulthood in a birth cohort. Community Dent Oral Epidemiol 2004;32:345-53.

18 Guan Y, Zeng X, Tai B, et al. Socioeconomic inequalities in dental caries among 5 -year-olds in four Chinese provinces. Community Dent Health 2015;32:185-9.

19 Wong HM, McGrath CPJ, King NM, et al. Oral health-related quality of life in Hong Kong preschool children. Caries Res 2011;45:370-6.

20 Lu HX, Tao DY, Lo ECM, et al. The 4th national oral health survey in the mainland of China: background and methodology. Chin J Dent Res 2018;21:161-5.

21 World Health Organization. Oral health surveys: basic methods. 5th edn. Geneva: World Health Organization, 2013.

22 Pedersen AB, Mikkelsen EM, Cronin-Fenton D, et al. Missing data and multiple imputation in clinical epidemiological research. Clin Epidemiol 2017;9:157-66.

23 White IR, Royston P, Wood AM. Multiple imputation using chained equations: issues and guidance for practice. Stat Med 2011;30:377-99.

24 MacDonald R. Overdispersion and poisson regression 2008;24:269-84.

25 Coxe S, West SG, Aiken LS. The analysis of count data: a gentle introduction to Poisson regression and its alternatives. J Pers Assess 2009;91:121-36.

26 Mackenbach JP, Kunst AE. Measuring the magnitude of socioeconomic inequalities in health: an overview of available measures illustrated with two examples from Europe. Soc Sci Med 1997:44:757-71.
27 Boston College Department of Economics. RIIGEN: Stata module to generate variables to compute the Relative Index of Inequality [program]. revised 21 Nov 2013 version 2013.

28 Kato H, Tanaka K, Shimizu K, et al. Parental occupations, educational levels, and income and prevalence of dental caries in 3-year-old Japanese children. Environ Health Prev Med 2017;22:80.

29 Peres MA, Macpherson LMD, Weyant RJ, et al. Oral diseases: a global public health challenge. Lancet 2019;394:249-60.

30 Armfield JM. Socioeconomic inequalities in child oral health: a comparison of discrete and composite area-based measures. $J$ Public Health Dent 2007;67:119-25.

31 Zhou Y, Chen DR, Zhi QH, et al. The prevalence and associated risk indicators of dental fluorosis in China: findings from the 4th national oral health survey. Chin J Dent Res 2018;21:205-11.

32 Jamieson LM, Armfield JM, Roberts-Thomson KF. Oral health inequalities among indigenous and nonindigenous children in the Northern Territory of Australia. Community Dent Oral Epidemiol 2006;34:267-76.

33 Shen $\mathrm{A}$, Zeng $\mathrm{X}$, Cheng $\mathrm{M}$, et al. Inequalities in dental caries among 12-year-old Chinese children. J Public Health Dent 2015;75:210-7.

34 Kragt L, Wolvius EB, Raat $\mathrm{H}$, et al. Social inequalities in children's oral health-related quality of life: the generation R study. Qual Life Res 2017;26:3429-37.

35 Lu HX, Wong MCM, Lo ECM, et al. Trends in oral health from childhood to early adulthood: a life course approach. Community Dent Oral Epidemiol 2011;39:352-60.

36 Slade GD, Sanders AE. Two decades of persisting income-disparities in dental caries among US children and adolescents. J Public Health Dent 2018;78:187-91.

37 Srisilapanan P, Nirunsittirat A, Roseman J. Trends over time in dental caries status in urban and rural Thai children. J Clin Exp Dent 2017;9:e1201.

38 Zhao Y, Zhang L, Fu Y, et al. Socioeconomic disparities in cancer treatment, service utilization and catastrophic health expenditure in China: a cross-sectional analysis. Int J Environ Res Public Health 2020;17 doi:10.3390/ijerph17041327

39 Zhang R, Chen Y, Liu S, et al. Progress of equalizing basic public health services in Southwest China- health education delivery in primary healthcare sectors. BMC Health Serv Res 2020;20:247.

40 Zhang Y, Li KY LECM, et al. Structural equation model for parental influence on children's oral health practice and status. BMC Oral Health 2020;20:1-10.

41 Gao X, Ding M, Xu M, et al. Utilization of dental services and associated factors among preschool children in China. BMC Oral Health 2020;20:1-10.

42 Pieper K, Dressler S, Heinzel-Gutenbrunner M, et al. The influence of social status on pre-school children's eating habits, caries experience and caries prevention behavior. Int $J$ Public Health 2012;57:207-15.

43 Armfield JM, Spencer AJ, Roberts-Thomson KF, et al. Water fluoridation and the association of sugar-sweetened beverage consumption and dental caries in Australian children. Am J Public Health 2013;103:494-500.

44 Borrell LN. Oral health inequities: an AJPH supplement to help close the gap. American Public Health Association, 2017.

45 Cheng ML, Xu MR, Xie YY, et al. Utilisation of oral health services and economic burden of oral diseases in China. Chin J Dent Res 2018;21:275-84

46 Harper HJ, Conicella ML, Cranston NC. The Aetna-NDA partnership for achieving racial and ethnic health equity. American Public Health Association, 2017. 